\title{
Modeling Thermal Effects on the Light vs. Current Characteristic of Gain-Guided Vertical-Cavity Surface-Emitting Lasers
}

\author{
J. Piprek, H. Wenzel and G. Sztefka
}

\begin{abstract}
Heating effects on the light power vs. current characteristic of planar top-emitting vertical-cavity laser diodes are analyzed by thermal, optical and electrical modeling. The twodimensional finite element thermal-electrical simulation considers the spatial current funneling in the top $p$-doped distributed Bragg reflector and a current density dependent resistivity of the hetero-barrier. Layered regions are described by anisotropic material parameters. Non-uniform refractive index temperature coefficients of the semiconductor materials are applied in the vertical optical modeling. The strongly inhomogeneous temperature distribution causes thermal increases of emission wavelength and reflectivity as well as decreases of threshold gain and external quantum efficiency with rising current. Finally, the calculated maximum light power is found to be decisively affected by the temperature coefficients of the refractive indices.
\end{abstract}

\section{INTRODUCTION}

V ERTICAL-CAVITY surface-emitting laser (VCSEL) diodes are a promising new generation of optical sources which may be utilized in future information processing and communication systems [1]. To support further VCSEL development, modeling efforts are needed to understand and limit several troublesome effects. One of these effects is the thermally induced decrease of output power with higher current seen in a roll-over of the light power vs. current (PI) characteristic. Recently, investigations of this behavior include PI-curve simulations for index-guided VCSELs with upper distributed Bragg reflectors (DBR) formed by etching [2]-[4]. In the present paper, a planar gain-guided top-emitting structure [5] is analyzed. Here, more attention has to be paid to thermal modeling than in the index-guided case because of the nonuniformity of heat generation and heat flux within the $p$-doped DBR. Such diodes are lasing in continuous wave $(\mathrm{CW})$ at up to $120^{\circ} \mathrm{C}$ ambient temperature [6], indicating a temperature rise in the active region of more than $100 \mathrm{~K}$ during room temperature $\mathrm{CW}$ operation. The heating within the $p$-DBR is even higher and is not without impact on the optical properties of the VCSEL. Our letter focuses on the reduction of threshold gain $g_{\text {th }}$ and external quantum efficiency $\eta_{\text {ext }}$ caused by thermally improved mirror reflectivity, and its consequences

Manuscript received September 27, 1993; revised November 19, 1993.

J. Piprek and H. Wenzel are with Humboldt Universität Berlin, Fachbereich Physik, Unter den Linden 6, 10099 Berlin, Germany. Piprek's present address is University of Delaware, Materials Science Program, Newark, DE 197163106.

G. Sztefka is with the Heinrich Hertz Institut für Nachrichtentechnik, Einsteinufer 37, 10587 Berlin, Germany.

IEEE Log Number 9215886.
Current through the graded GaAs/AlAs Interface (p-DBR) solving Schrödinger-Poisson-System

$\rho(j, T)$

3D Current Density and Temperature Distribution in VCSEL solving Flux Equations by Finite Element Method

$$
T(r, z, I)
$$

1D Electromagnetic Field in VCSEL at Threshold solving Maxwells Equations by Transfer Matrix Method

$g_{t h}(I), \eta_{e x t}(I), \lambda_{0}(I)$

Threshold Current for Strained Quantum Wells calculating Gain Function and Recombination Rates

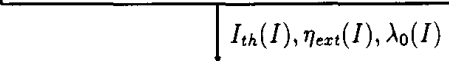

Light Power vs. Current $P(I)=\eta_{\text {ext }}\left(I-I_{t h}\right) h c / e \lambda_{0}$

Fig. 1. Schematic view of the VCSEL modeling steps as explained in the text $(h c / e=1241 \mathrm{~V} \mathrm{~nm})$.

for the PI characteristic. This particular effect has not been reported yet and is of general interest for gain-guided and index-guided VSCEL structures. The modeling procedure ${ }^{1}$ is given schematically in Fig. 1.

Our device example is a three-quantum-well $\mathrm{In}_{0.2} \mathrm{Ga}_{0.8} \mathrm{As} /$ GaAs top-emitting VCSEL with an emission wavelength of typically $\lambda_{0}=980 \mathrm{~nm}$. It contains a stepped-index cavity (Al fraction varied from 0.125 to 0.5 ) plus GaAs spacer sandwiched between the 19.5 period top DBR ( $p$-doping: 4 $\times 10^{18} \mathrm{~cm}^{-3}$ ) and the 23.5 period bottom DBR ( $n$-doping: $2 \times 10^{18} \mathrm{~cm}^{-3}$ ) on $300 \mathrm{~nm}$ GaAs substrate. To reduce series resistance, the interfaces of the AlAs/GaAs $p$-DBR stack include $4 \mathrm{~nm}$ thick $\mathrm{Al}_{0.5} \mathrm{Ga}_{0.5} \mathrm{As}$ interlayers and are $p$ - $\delta$-doped as described in [7]. Within the top DBR, proton implantation provides lateral current funneling with $6 \mu \mathrm{m}$ minimum diameter, $2.5 \mu \mathrm{m}$ below the top surface.

Excessive Joule heating within the $p$-DBR is considered the main heat source due to potential barriers at the in-

'Further details of the model will be published elsewhere. 
terfaces of the semiconductor lattice. The resistivity of the stepped AlAs/GaAs interface has been calculated by solving the Schrödinger-Poisson-System [8], but the unknown distribution of the $p$-dopant Beryllium [7] remains a major uncertainty. Tunneling currents depend crucially on the shape of the potential spike and therefore on the doping profile. Thus, measurements on separated $p$-DBR stacks were performed [9], yielding the resistivity vs. current density function $\varrho(j)=1117 \Omega \mathrm{cm}\left(j / \mathrm{A} \mathrm{cm}^{-2}\right)^{-0.792}$. This equation describes the vertical $p$-DBR resistivity, whereas the lateral resistivity paralle]. to the interfaces is much lower.

For the thermal-electrical simulation, the three-dimensional finite element code ANSYS [10] is employed, permitting anisotropic material parameters. First, the spatial current density distribution $j(r, z)(r, z$-cylinder coordinates) is calculated self-consistently, which determines the thermal power density distriburion $\varrho j^{2}$ within the $p$-DBR. Besides this main heat source, the contact resistance, active region heating, and internal light absorption are taken into account by solving the thermal conduction equation. Herein, the thermal conductivity $\kappa$ of layered structures depends on the heat flux direction. In general, a simple connection of serial or parallel thermal sheet resistances can be assumed for the calculation of vertical and lateral conductivities $\kappa_{\mathrm{v}}$ and $\kappa_{\mathrm{l}}$, respectively. Especially for the AlAs/GaAs lattice, a vertical value of $\kappa_{\mathrm{v}} \approx 0.3 \mathrm{~W} / \mathrm{cm} \mathrm{K}$ has been measured [11], which is less than half of $\kappa_{1}=0.69$ $\mathrm{W} / \mathrm{cm} \mathrm{K}$. Thus, most of the heat generated in the $p$-DBR flows first in the lateral direction to reach the bottom contact of the substrate (heat sink $T=300 \mathrm{~K}$ ). With high values of the current $I$, the resulting temperature distribution $T(r, z)$ shows a temperature rise of more than $100 \mathrm{~K}$ within the optical resonatur. Fig. 2 displays solutions $T(0, z)$ along the resonator axis of our device. Each current value $I$ is connected with a certain temperature distribution, leading to laser threshold parameters as function of $I$.

The next step in the simulation procedure is the vertical optical modeling of the electromagnetic field distribution within the VC.SEL resonator at laser threshold. For this purpose, Maxwells Equations are solved by the transfer matrix method (TMM). Here, the propagation of forward and backward traveling waves through the layers is performed by matrix multiplications. The tangential field components must be continuous at the interfaces and, with a further inverse matrix multiplication, yield the wave amplitudes in the next layer [12]. Recursive calculations for all layers give the overall Iransfer matrix of this device. The laser condition (no incoming waves from outside) leads to an eigenvalue problem in the complex plane. From the real and imaginary parts of the eigenvalue, $\lambda_{0}$ and $g_{\mathrm{th}}$ can be found. The external quanturr efficiency $\eta_{\text {ext }}=\left|E_{\text {out }}\right|^{2} / g_{\mathrm{th}} d_{\mathrm{QW}} n_{\mathrm{QW}}\left|E_{\mathrm{QW}}\right|^{2}$ is then obtained from the intensity ratio $\left|E_{\text {out }}\right|^{2} /\left|E_{\mathrm{QWW}}\right|^{2}$ of the emitted wave and the average electrical field within the quanturn wells (QWs) with $d_{\mathrm{QW}}$ and $n_{\mathrm{QW}}$ denoting total thickness and refractive index of the QWs, respectively. For our device, room temperature values of $\lambda_{0}=981 \mathrm{~nm}, g_{\mathrm{th}}=$ $490 \mathrm{~cm},^{-1}$ and $\eta_{\text {ext }}=0.21$ are calculated.

Heating effects on the optical behavior are connected with the temperature dependence of the refractive index $n(\Delta T)=$

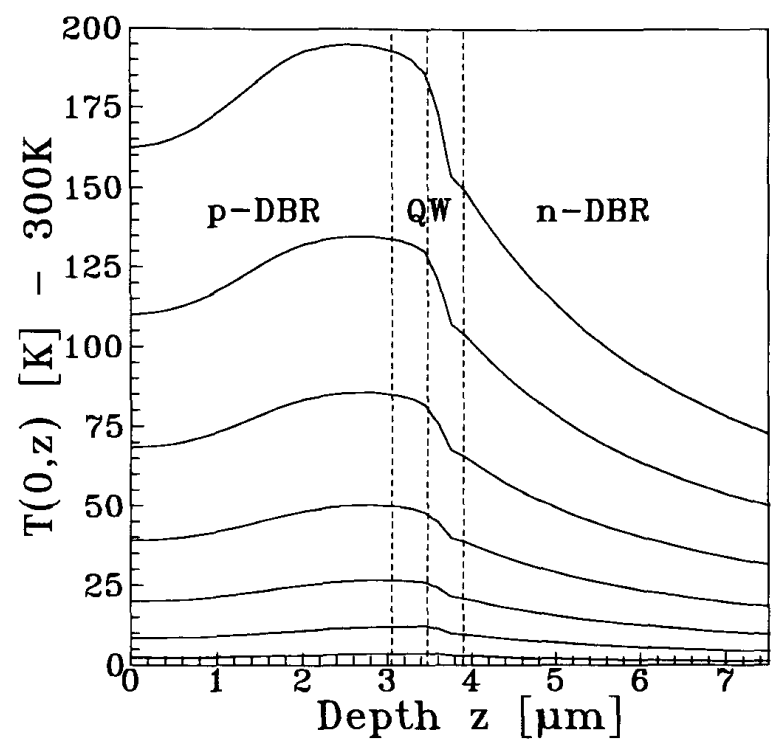

Fig. 2. Calculated temperature distribution $T(0, z)$ along the axis of the VCSEL resonator with ascending current values $I[\mathrm{~mA}]: 1.5,4.4,8.8,14.7$, $22.1,30.8,40.6$. The steeper slope within the cavity is due to the small thermal conductivity of the $\mathrm{Al}_{0.5} \mathrm{Ga}_{0.5}$ As confinement layer.

$n_{0}+\beta \Delta T$. The temperature coefficient $\beta$ of $\mathrm{GaAs}$ is known to be about $4 \times 10^{-4} \mathrm{~K}^{-1}$ [13], for AlAs a value of about $1 \times 10^{-4} \mathrm{~K}^{-1}$ can be deduced from measurements [14]. The laser photon energy is closer to the band gap energy of GaAs, the refractive index of GaAs is therefore more influenced by the thermal band gap shrinkage than in AlAs. In the case of ternary $\mathrm{Al}_{x} \mathrm{Ga}_{1-x} \mathrm{As}$, no measurements can be found. Using the model in [15], theoretical calculations confirm the reported margin values and yield $\beta=2 \times 10^{-4} \mathrm{~K}^{-1}$ for $x=0.5$. Additionally, we include thermal expansion of the sheet thicknesses $d$ with the linear expansion coefficient $6 \times 10^{-6} \mathrm{~K}^{-1}$ of our semiconductor materials [16]. According to this value, a $100 \mathrm{~K}$ temperature rise generates an increase in layer thickness of only $0.06 \%$.

For the device example, the thermal shifts of the TMM results $g_{\mathrm{th}}, \eta_{\text {ext }}$ and $\lambda_{0}$ are shown in Fig. 3, considering the axial temperature distributions in Fig. 2. The dependency $n(T)$ dominates these effects, whereas $d(T)$ is without remarkable influence. The emission wavelength $\lambda_{0}$ rises with increasing temperature, due to an enlarged optical length of the cavity. But the threshold gain $g_{\mathrm{th}}$ as well as the external quantum efficiency $\eta_{\text {ext }}$ are substantially reduced by the heating. In the lower index material (AlAs, $n_{0}=2.96$ ) the increase rate $\beta$ is smaller than in $\operatorname{GaAs}\left(n_{0}=3.52\right)$. Thus, the DBR reflectivity $\left(n_{\mathrm{GaAs}}-n_{\mathrm{AlAs}}\right)^{2} /\left(n_{\mathrm{GaAs}}+n_{\mathrm{AlAs}}\right)^{2}$ is thermally improved, more radiation is kept inside and less gain is necessary for lasing. This is in contrast to the computed results in [4], where $\beta=4 \times 10^{-4} \mathrm{~K}^{-1}$ is assumed uniform in all materials and, consequently, the reflectivity decreases with temperature, resulting in higher values of $g_{\mathrm{th}}$ and $\eta_{\text {ext }}$. Taking the temperature $T_{\mathrm{QW}}$ of the QWs as abscissa in Fig. 3, nearly linear temperature dependencies of these three parameters 


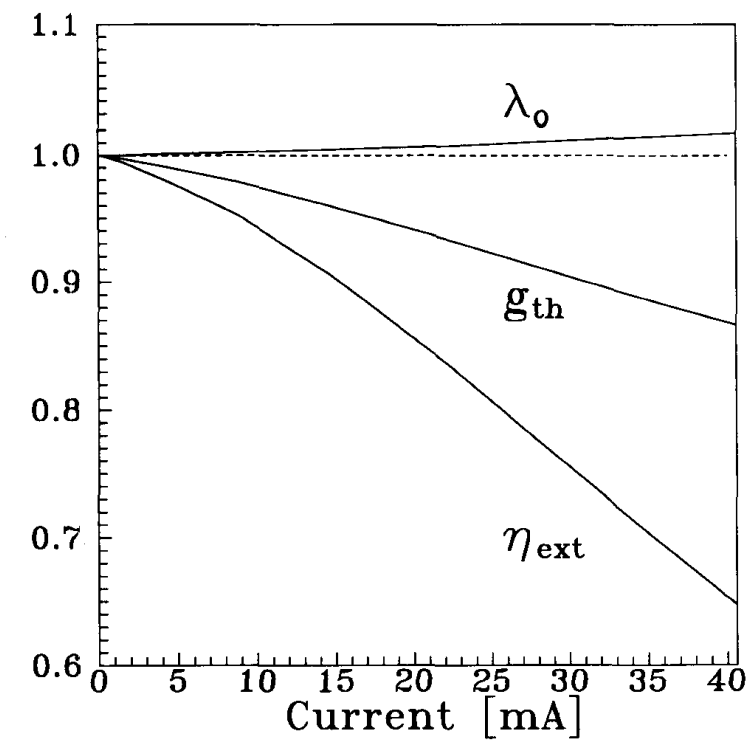

Fig. 3. Relative changes of emission wavelength $\lambda_{0}$, threshold gain $g_{\mathrm{th}}$, and external quantum efficiency $\eta_{\text {ext }}$ as function of the current $I$, calculated according to Fig. 2.

occur and the relative change per $100 \mathrm{~K}$ is $0.9 \%,-7.4 \%$ and $-19.5 \%$ for $\lambda_{0}, g_{\mathrm{th}}$ and $\eta_{\text {ext }}$, respectively. The first value is in good agreement with the measured thermal red-shift of the lasing wavelength $(0.9 \%$ [3], $0.7 \%$ [5]). PI measurements at different heat sink temperatures confirm a reduction of $\eta_{\text {ext }}$ with rising temperature [5], [6]. In principle, $g_{\text {th }}$ could be calculated from the measured threshold current [3], but with theoretical parameters which are too uncertain to give an unambiguous verification of the simulated $g_{\mathrm{th}}$ lowering.

Lateral waveguiding is not included in our model, but thermal lensing [17] would even strengthen the $g_{\mathrm{th}}$ reduction. The increase of absorption with temperature weakens the $g_{\text {th }}$ drop, but its quantitative consideration requires more experimental investigations.

The next modeling step is the calculation of the gain function $g\left(N, \lambda_{0}, T_{\mathrm{QW}}\right)$ to derive the threshold carrier density $N_{\mathrm{th}}$ from the already known parameters $g_{\mathrm{th}}, \lambda_{0}$ and $T_{\mathrm{QW}}$. Doing this, we assume direct transitions between parabolic bands with a Lorentzian broadening function and include the compressive strain within the QWs [18]. The temperature dependence of the gain is due to the temperature dependencies of gap energy and quasi-Fermi levels. The threshold density leads to the threshold current $I_{\mathrm{th}}$, which is calculated by adding the contributions of Shockley-Read-Hall recombination, spontaneous emission, Auger recombination and vertical leakage (recombination within the confinement layers [18]). Only the last two recombination currents are computed to rise strongly enough with temperature to cause the PI rollover. For the present example, room temperature values of $N_{\text {th }}=1.6 \times 10^{18} \mathrm{~cm}^{-3}$ and $I_{\text {th }}=2.2 \mathrm{~mA}$ are obtained. Due to the thermally increased mismatch between $\lambda_{0}(I)$ and the wavelength of maximum gain, $N_{\mathrm{th}}(I)$ is rising despite the $g_{\mathrm{th}}$ reduction. At $I=40 \mathrm{~mA}$ the threshold current

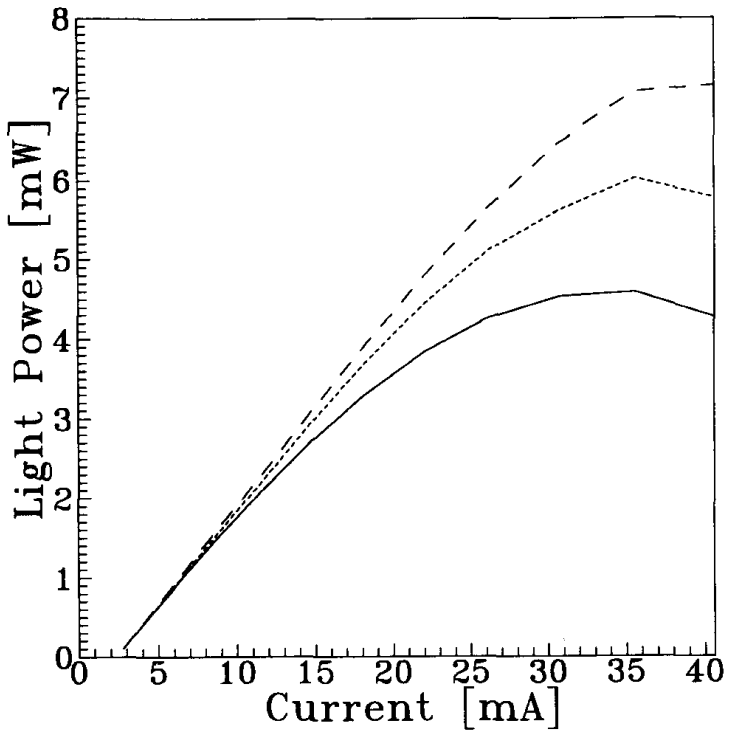

Fig. 4. Calculated light power vs. current characteristics assuming different temperature coefficients $\beta\left[10^{-4} \mathrm{~K}^{-1}\right]$ of the refractive index in $\mathrm{Al}_{x} \mathrm{Ga}_{1-x}$ As (solid: $4(x=0), 2(x=0.5), 1(x=1)$, dashed: 4 , dotted: 0$)$.

$I_{\mathrm{th}}(I)$ is almost seven times higher than at room temperature.

With $\eta_{\text {ext }}(I), \lambda_{0}(I)$ and finally $I_{\mathrm{th}}(I)$, all quantities are gathered to calculate the PI characteristic (see Fig. 1). Using the previous results, a maximum output power of $P_{\max }=$ $4.6 \mathrm{~mW}$ occurs at $I=36 \mathrm{~mA}$ (solid curve in Fig. 4). This PI curve is compared to the assumption of an uniform temperature coefficient $\beta=4 \times 10^{-4} \mathrm{~K}^{-1}$ in all materials (dashed curve in Fig. 4) increasing $P_{\max }$ by $55 \%$ due to the rising external quantum efficiency. In the case without any temperature dependency of the refractive index $\beta=0$ (dotted curve in Fig. 4) a medium value of $P_{\max }$ is obtained because the thermal shifts of $\eta_{\mathrm{ext}}, g_{\mathrm{th}}$ and $\lambda_{0}$ are vanishing. For comparison with actual devices, the theoretical PI curve has to be fitted to the measurement under special consideration of the particular leakage current [3].

In summary, with emphasis on thorough thermal modeling a numerical procedure has been developed to simulate the light power vs. current characteristic of planar gain-guided vertical-cavity top surface-emitting laser diodes. Applying the proper refractive index temperature coefficient of the different semiconductor materials results in thermally caused reductions of threshold gain and external quantum efficiency with rising current. These shifts are shown to be essential for computing the maximum output power of the laser diode.

\section{REFERENCES}

[1] K. Iga and F. Koyama, "Vertical cavity surface emitting lasers and arrays," in: Surface Emitting Semiconductor Lasers and Arrays, ed. G. A. Evans and J. M. Hammer, Academic Press, San Diego, 1993, pp. 71-117.

[2] J. W. Scott, S. W. Corzine, D. B. Young and L. A. Coldren, "Modeling the current to light characteristics of index-guided vertical-cavity 
surface-emitting lasers," Appl. Phys. Lett., vol. 62, pp. 1050-1052, March 1993

[3] J. W. Scott, R. S. Geels, S. W. Corzine and L. A. Coldren, "Modeling temperature effects and spatial hole burning to optimize vertical-cavity surface-emitting laser performance," IEEE J. Quant. El., vol. 29, pp. 129.5-1308, May 1993.

[4] R. Michalzik and K. J. Ebeling, "Modeling and design of protonimplanted ultralow-threshold vertical cavity laser diodes," IEEE $J$. Quant. El., vol. 29, pp. 1963-1974, June 1993

[5] R. A. Morgan, L. M. F. Chirovsky, M. W. Focht, G. Guth, M. T. Asom, R. E. Leibenguth, K. C. Robinson, Y. H. Lee and J. L. Jewell, "Progress in planarized vertical cavity surface emitting laser devices and arrays," in: Devices for Optical Processing, SPIE Proc. vol. 1562, pp. 149-158, July 1991.

[6] J. M. Catchmark, R. A. Morgan, K. Kojima, R. E. Leibenguth, G. D. Guth, M. W. Focht, M. T. Asom, L. C. Luther and G. P. Przybylek, "High temperature $\mathrm{cw}$ operation of vertical-cavity top surface-emitting lasers," Conf. Lasers and Electroopt. CLEO'93, Baltimore, May 1993, pp. $138-139$.

[7] A. Paraskevopoulos, H. Künzel, P. Harde, J. Böttcher, R. Bochnia and S. Gramlich, "Low temperature growth of Be-doped GaAs/AlAs distributed Bragg reflector structures with $\delta$-doped transition layers," Conf. Lasers and Electroopt. CLEO'93, Baltimore, May 1993, pp. $80-83$.

[8] J. Fjprek and K. J. Friedland, unpublished.
[9] A. Paraskevopoulos, unpublished.

[10] ANSYS Release 5.0 (1993) by Swanson Analysis Systems, Inc., Houston, PA, U.S.A

[11] T. Yao, "Thermal properties of AlAs/GaAs superlattices," Appl. Phys. Lett., vol. 51, pp. 1798-1800, Nov. 1987.

[12] J. Chilwell and I. Hodgkinson, "Thin-film field-transfer matrix theory of planar multilayer waveguides and reflection from prism-loaded waveguides," J. Opt. Soc. Am. A, vol. 1, pp. 742-753, 1984.

[13] H. C. Casey and M. B. Panish, Heterostructure Lasers, Part A, Academic Press, New York, 1978, p. 31

[14] H. G. Grimmeiss and B. Monemar, "Temperature dependence of the refractive index of AlAs and AlP," phys. stat. sol. (a) vol. 5, pp. 109-114, 1971.

[15] B. Jensen and W. D. Jensen, "The refractive index near the fundamental absorption edge in $\mathrm{Al}_{x} \mathrm{Ga}_{1-x} \mathrm{As}$ ternary compound semiconductors," IEEE J. Quantum Electron., vol. 27, pp. 40-45, Jan. 1991.

[16] S. Adachi, Data in Science and Technology: Semiconductors, Group IV Elements and III-V Compounds, Springer, New York 1991.

[17] G. Hasnain, K. Tai, L. Yang, Y. H. Wang, R. J. Fischer, J. D. Wynn, B. Weir, N. K. Dutta and A. Y. Cho, "Performance of gain-guided surface emitting lasers with semiconductor distributed bragg reflectors," IEEE J. Quant. Electr. vol. 27, pp. 1377-1385, June 1991.

[18] H. Wenzel and H.-J. Winsche, "A model for the calculation of the threshold current of SCH-MQW-SAS lasers," phys. stat. sol. (a) vol. 120, pp. 661-673, 1990. 